\title{
Turbulenzen im Kinderleben. Mütterliche Partnerschaftsbiographien und multiple Übergänge nach elterlicher Trennung
}

\author{
Turbulences in children's lives. Mothers' union histories and multiple \\ transitions after parental separation
}

\begin{abstract}
Zusammenfassung:
Nach einer elterlichen Trennung gilt das Erleben multipler Übergänge als Risikofaktor für Kinder. Wir analysieren erstmals für Österreich im Zeitverlauf, wie viele Übergänge, definiert als neuerliche mütterliche Partnerbeziehungen oder Trennungen, Kinder erleben und von welchen Determinanten dies beeinflusst wird. Die Analysen erfolgen mittels multinomialer logistischer Regression auf Basis der österreichischen Daten des Fertility and Family Survey 1995/96 und des Generations and Gender Survey 2008/09. Die Ergebnisse zeigen, dass innerhalb von acht Jahren nach der elterlichen Trennung jeweils rund 50\% der Kinder keine und rund $40 \%$ genau eine neue Kohabitation (Lebensgemeinschaft oder Ehe) der Mutter erleben, zumeist in den ersten vier Jahren nach der elterlichen Trennung. Fast jedes siebte Kind mit getrennten Eltern (13\%) macht die Erfahrung multipler Übergänge. Erklärende Faktoren sind niedriges Alter der Mutter zum Zeitpunkt der Trennung, geringes mütterliches Bildungsniveau sowie niedriges Alter des jüngsten Kindes. Des Weiteren zeigt sich ein deutlicher Effekt des Trennungsjahres: die Wahrscheinlichkeit für das Erleben multipler Übergänge ist im Zeitverlauf markant angestiegen.
\end{abstract}

Schlagwörter: Scheidung, Trennung, Kinder, Familienstruktur, multiple Übergänge, Partnerschaftsbiographie

\begin{abstract}
:
Experiencing multiple transitions after a parental separation was identified as an important risk factor for children. We investigate for the first time for Austria, how many transitions (i.e. changes in maternal partnership arrangements) children experience and what determines their number. The analyses are based on the Austrian Fertility and Family Survey 1995/96 and the Generations and Gender Survey 2008/09 using a multinomial logistic regression model. The results show that within eight years after parental separation, about $50 \%$ of children experience no new cohabitation or marriage of their mother and about $40 \%$ experience exactly one. For the majority of children, these changes happen within four years after their parents' separation. Almost every seventh child with separated parents $(13 \%)$ experiences multiple transitions. Children with a low-level educated mother and those whose mother is young at the time of separation face a particularly high risk, as well as those living in families with a low age of the youngest child. Moreover, a strong effect of the year of separation could be detected: the probability of experiencing multiple transitions increases strongly over time.
\end{abstract}

Key words: divorce, separation, children, family structure, multiple transitions, partnership history 


\section{Einleitung}

Trennung oder Scheidung der Eltern bedeutet für Kinder eine fundamentale Veränderung ihrer Lebensbedingungen. Forschungsergebnisse zu den Konsequenzen einer Scheidung für Kinder weisen auf unterschiedliche negative Auswirkungen hin, welche durch moderierende Faktoren beeinflusst werden (Amato 2000; Amato et al. 2011). Solche Faktoren sind neben individuellen, interpersonellen und strukturellen Ressourcen auch die Verläufe und Dynamiken, welche einer elterlichen Trennung folgen. Wird diese nicht statisch im Sinn eines einmaligen Übergangs konzipiert, sondern dynamisch betrachtet, so kann sie der Auftakt für eine Reihe weiterer familienstruktureller Veränderungen sein, wie Kohabitation eines Elternteils mit einem neuen Partner, Wiederverheiratung oder weitere Trennungen.

Die auf eine elterliche Trennung folgenden familienstrukturellen Dynamiken stehen im Mittelpunkt dieses Beitrags. Im englischsprachigen Raum gibt es bereits seit den 1990er Jahren eine intensive Beschäftigung mit Familienverläufen nach einer elterlichen Trennung und deren Auswirkungen auf Kinder. Die Ergebnisse weisen darauf hin, dass vor allem das Erleben multipler Transitionen mit negativen Auswirkungen für Kinder einhergeht (Amato/Sobolewski 2001; Cavanagh/Huston 2006; Fomby/Cherlin 2007; Osborne/McLanahan 2007; Kiernan/Mensah 2010). Inwieweit diese vorwiegend auf USamerikanischen Studien basierenden Befunde auf den deutschsprachigen Raum übertragen werden können, ist unklar. Für Deutschland liegen vereinzelt deskriptive ältere Befunde zur familienstrukturellen Entwicklung nach einer Trennung vor (Stegmann 1997; Alt/Bender 1998). Einige rezente Arbeiten greifen einzelne Aspekte auf und unterstreichen damit die Relevanz und Aktualität des Themas (Wendt/Walper 2007; Feldhaus/ Huinink 2011; Jaschinski 2011; Bastin 2012). In Österreich wurden kindliche Lebensverläufe nach einer elterlichen Trennung bislang nicht systematisch analysiert. Damit bleibt völlig unklar, wie viele familienstrukturelle Veränderungen Kinder nach einer elterlichen Trennung erleben, wann diese stattfinden und von welchen Determinanten sie bedingt werden.

Der vorliegende Beitrag setzt sich zum Ziel, diese Forschungslücke zu schließen, und fokussiert auf mütterliche Partnerschaftsverläufe. ${ }^{1}$ Wir analysieren erstmals detailliert im Zeitverlauf, wie viele Übergänge Kinder in den ersten acht Jahren nach einer elterlichen Trennung ${ }^{2}$ erleben, wann diese stattfinden und in welchen familialen Lebensformen Kinder nach einer elterlichen Trennung leben. Darüber hinaus wird eruiert, welche Determinanten die Anzahl der familialen Übergänge erklären und die Wahrscheinlichkeit, multiple Übergänge zu erleben, reduzieren können. Übergänge ${ }^{3}$ werden definiert als Veränderungen in der mütterlichen Partnerschaftsbiographie, welche nach der elterlichen Trennung erfolgen. Als Übergänge gelten Heirat bzw. Kohabitation sowie Scheidung bzw.

1 Da ein Großteil aller Kinder nach der elterlichen Trennung überwiegend bei der Mutter lebt, konzentrieren wir uns auf Veränderungen in der mütterlichen Partnerschaftsbiographie.

2 Berücksichtigt werden sowohl Kinder, deren Eltern vor der Trennung verheiratet waren als auch jene, deren Eltern unverheiratet waren. Aus Gründen der Lesbarkeit verwenden wir den Terminus Trennung, welcher Scheidung inkludiert.

3 Die Begriffe Transition und Übergang werden deckungsgleich verwendet. 
Trennung der Mutter. Die Datenbasis bilden der österreichische Fertility and Family Survey 1995/96 sowie der Generations and Gender Survey 2008/09.

Österreich repräsentiert hinsichtlich familienpolitischer und -rechtlicher Aspekte ein konservatives wohlfahrtsstaatliches Regime (Esping-Andersen 1990; Pfau-Effinger/Lewis 1995), charakterisiert durch relativ gut ausgebaute wohlfahrtsstaatliche Leistungen (hohe finanzielle Transferleistungen, lange Elternkarenz), eine Akzentsetzung zugunsten von Ehen sowie eine starke Ausrichtung am (modifizierten) männlichen Ernährermodell (Haas 2009; Hopf 2011; Zartler 2012a). Alleinerzieherinnen weisen eine stark erhöhte Armutsgefährdung auf, die durch staatliche Transferleistungen nur zum Teil abgefedert werden kann (Zartler/Beham 2011). Die österreichische Gesamtscheidungsrate liegt mit 43\% im oberen europäischen Mittelfeld. Das Scheidungsrisiko aus Kindersicht, d.h. die Wahrscheinlichkeit für ein ehelich geborenes Kind, bis zum 18. Geburtstag eine elterliche Scheidung zu erleben, liegt bei 20\% (Statistik Austria 2013a). Diese offiziellen Daten unterschätzen allerdings die Gesamtzahl der Kinder mit Trennungserfahrungen, da Trennungen nichtehelicher Lebensgemeinschaften nicht registriert werden. Vier von zehn österreichischen Kindern werden unehelich geboren ${ }^{4}$ (Statistik Austria 2012: 24), und Kohabitationen haben eine hohe gesellschaftliche Akzeptanz (Zartler 2013). Werden sowohl das Scheidungs- als auch das Trennungsrisiko aus Kindersicht berücksichtigt, so zeigt sich eine Wahrscheinlichkeit von 30\%, bis zum 18. Geburtstag eine elterliche Scheidung oder Trennung zu erleben (eigene Berechnungen auf Basis des Generations and Gender Survey 2008/09; bezogen auf Kinder, die zum Zeitpunkt der Umfrage 18-25 Jahre alt waren).

Nach einer elterlichen Trennung lebt der Großteil der Kinder vorwiegend bei der Mutter. ${ }^{5}$ Rund $13 \%$ aller Kinder unter 18 Jahren leben in einer Ein-Eltern-Familie, davon $92 \%$ bei der Mutter und $8 \%$ beim Vater; $6 \%$ aller Kinder unter 18 Jahren leben gemeinsam mit einem Stiefelternteil im Haushalt, davon $89 \%$ bei ihrer leiblichen Mutter und 11\% beim leiblichen Vater (Statistik Austria 2013b: 68, 83).

\section{Multiple Übergänge im Kinderleben. Theoretische Grundlagen und bisher vorliegende Ergebnisse}

Forschungsarbeiten zu multiplen Transitionen haben seit den 1990er Jahren stark an Einfluss gewonnen (Cowan/Hetherington 1991; Amato 1993; Aquilino 1996; Flowerdew/ Neale 2003; Amato 2010). Sie gehen davon aus, dass eine umfassende Analyse der Auswirkungen von Trennungen es erfordert, auch die familiendynamische Entwicklung danach ins Blickfeld zu nehmen. Abhängig von Anzahl und Ausgestaltung der familienstrukturellen Veränderungen werden instabile und stabilisierte Nachscheidungsfamilien unterschieden (Sun/Li 2008).

4 Rund die Hälfte aller unehelich geborenen Kinder wird bis zum 18. Geburtstag durch eine nachfolgende Eheschließung der Eltern legitimiert (Statistik Austria 2011, 121f.).

5 Die Mutter bleibt in neun von zehn Fällen auch bei Obsorge beider Elternteile (gemeinsames Sorgerecht) der Residenz-Elternteil, d.h. jener Elternteil, bei dem das Kind vorwiegend lebt (Figdor et al. 2006). 
Der Begriff Transition bzw. Übergang wird unterschiedlich definiert, beispielsweise als jegliche Veränderung in der personellen Zusammensetzung des Haushalts aufgrund von Scheidung, Trennung, Tod oder Hinzukommen eines neuen Partners (Fomby/Cherlin 2007; Feldhaus et al. 2011), als Zustandswechsel zwischen unterschiedlichen Familienkonstellationen (Aquilino 1996; Feldhaus/Huinink 2011) oder als Veränderung des mütterlichen Partnerschaftsstatus (Osborne/McLanahan 2007). Nur wenige Studien inkludieren Beziehungen, in welchen der (neue) Partner nicht im selben Haushalt lebt (Osborne/ McLanahan 2007; Kiernan et al. 2011; Bastin 2012).

Aufgrund dieser divergierenden Definitionen ist die Befundlage zur Häufigkeit multipler Transitionen nicht eindeutig. So unterscheiden sich die Angaben danach, ob die Trennung der Referenzbeziehung (zumeist jene der leiblichen Eltern) bereits als erste Transition definiert wird. Aquilino (1996) zeigte, dass ein Fünftel der Kinder insgesamt (also die Trennung der leiblichen Eltern eingeschlossen) drei oder mehr Veränderungen der Familienform erlebt. In der Untersuchung von Amato und Sobolewski (2001) erlebten 11\% der Kinder zwei oder mehr Übergänge nach einer elterlichen Scheidung. In Deutschland erlebten nach den Berechnungen von Feldhaus et al. (2011) 11\% der Kinder der Geburtskohorte 19711973 während ihrer Kindheit genau einen Wechsel in der personellen Zusammensetzung des Haushalts (durch Scheidung, Trennung, Tod oder Hinzukommen eines neuen Partners), 4\% erlebten zwei und 0,7\% drei und mehr Übergänge. Darüber hinaus ist zu berücksichtigen, dass unter Umständen zunächst keine Trennung stattfindet, sondern manche Mütter von Geburt ihrer Kinder an alleine leben und/oder keine Partnerbeziehung haben. Bastin (2012) zeigte für die Lebensverläufe dieser Mütter, dass spätestens fünf Jahre nach der Geburt des ersten Kindes die Hälfte mit einem Partner (häufig der Vater des Kindes) zusammengezogen ist und dass Mütter, die bei der Geburt des Kindes einen Partner mit eigenem Haushalt haben (Living Apart Together; LAT), mit höherer Wahrscheinlichkeit bereits früh Trennungen und multiple Partnerschaftsübergänge erleben.

Mehrere Studien aus dem englischen Sprachraum konnten zeigen, dass multiple Transitionen mit einer Reihe negativer Auswirkungen für Kinder einhergehen, so beispielsweise mit Verhaltensproblemen (Cavanagh/Huston 2006; Osborne/McLanahan 2007; Kiernan et al. 2011), externalisierendem Verhalten (Fomby/Cherlin 2007; Kiernan/Mensah 2010), schlechteren schulischen und kognitiven Leistungen (Aquilino 1996; Hill et al. 2001) sowie geringerem emotionalen Wohlbefinden (Kiernan/Mensah 2010; Waldfogel et al. 2010). Amato/Sobolewski (2001) wiesen nach, dass negative Auswirkungen auf das kindliche Wohlbefinden besonders dann auftreten, wenn nach der elterlichen Trennung zwei oder mehr weitere Übergänge erlebt werden.

Diese negativen Auswirkungen multipler Transitionen werden häufig aus einer stresstheoretischen Perspektive erklärt (Acock/Demo 1994; Aquilino 1996; Amato 2000). Demnach gehen Veränderungen im Partnerschaftsstatus mit einer Erhöhung des Stressniveaus für Kinder, Eltern und neue Partner einher, da sich das Alltagsleben verändert, neue Routinen und Regeln entwickelt werden müssen und es zu einer Konkurrenz um Aufmerksamkeit, Zeit und Zuneigung kommen kann. Da Stress aus unterschiedlichen Transitionen kumuliert (Rutter 1981), ist es wahrscheinlich, dass Kinder, die mehrere Übergänge erfahren, eher negative Auswirkungen erleben als Kinder, welche keine oder lediglich eine Transition erleben. Multiple Transitionen in der eigenen Beziehungsbiographie haben auch einen negativen Einfluss auf Wohlbefinden und Gesundheit der El- 
tern, und zwar vor allem der Mütter, und können dadurch indirekt auf die Kinder wirken (Cavanagh/Huston 2006; Osborne/McLanahan 2007; Cooper et al. 2009).

Aus ressourcentheoretischer Sicht können die negativen Effekte multipler Übergänge mit Veränderungen der familialen (finanziellen, zeitlichen und sozialen) Ressourcen und einer daraus resultierenden Benachteiligung von Kindern erklärt werden. Beispiele sind negative Zusammenhänge zwischen neuerlichen Trennungen und ökonomischer Situation (Sun/Li 2008; Kiernan/Mensah 2010) oder die typischerweise sinkende Kontakthäufigkeit zwischen Kind und nicht-residenziellem Elternteil (zumeist der leibliche Vater), wenn ein Stiefvater hinzukommt oder die räumliche Entfernung sich nach Übergängen vergrößert (Juby et al. 2007; Jensen 2009; Tach et al. 2010).

Zentral für die Analyse von Übergängen sind theoretische Erklärungen zu Paarbildungsverhalten und Partnerschaftsverläufen, die überwiegend auf Modellen des rationalen Handelns basieren (Klein 2001; Lenz 2006). Grundlage ist häufig die mikro-ökonomische Marital Search Theory („Partnersuch-Theorie“) ${ }^{6}$ (Oppenheimer 1988), welche auch Determinanten der Partnerschaftsverläufe Alleinerziehender erklären kann (Edin/Kefalas 2005; Bastin 2012). Sie geht davon aus, dass die Partnersuche der Maximierung von Ressourcen dient. Dauer und Erfolg des Suchprozesses werden demnach von bestimmten Charakteristika der suchenden Person (z.B. Alter, Bildung, Verantwortung für Kinder), strukturellen Mechanismen des Partnermarktes sowie den relativen Suchkosten (Zeit, Geld, emotionales Engagement) beeinflusst. Es wird angenommen, dass die ökonomische Dringlichkeit, eine Partnerbeziehung einzugehen, für Alleinerziehende besonders relevant ist, da diese von einem zusätzlichen Einkommen bzw. einer zusätzlichen Betreuungsperson besonders profitieren (sofern Ressourcen innerhalb der Partnerbeziehung aufgeteilt werden) (Graefe/Lichter 2007). Des Weiteren wird vorausgesetzt, dass bei starker ökonomischer Dringlichkeit die Ansprüche an potentielle Partner verringert werden, um so den Pool an möglichen Partnern vergrößern und die Dauer der (kostenintensiven) Partnersuche verkürzen zu können.

Diese Überlegungen implizieren, dass für Frauen mit geringer Humankapitalausstattung (z.B. niedrige Bildung) der Übergang zu einer neuen Partnerschaft eher erwartet werden kann, da ihr ökonomischer Druck, einen finanziell entlastenden Partner zu finden, höher ist. Andererseits ist ihre Attraktivität auf dem Partnermarkt aber aufgrund der wenigen tauschbaren ökonomischen Ressourcen gering (Oppenheimer 1988), was erklären könnte, warum ökonomisch schlecht gestellte Alleinerziehende langsamer in andere Lebensformen wechseln (Fux 2011). Einige Arbeiten zeigen dementsprechend, dass die Neigung zur Paarbildung nach einer Trennung mit steigendem Bildungsniveau ansteigt, was damit erklärt werden kann, dass höhere Bildung mit höherer Attraktivität auf dem Partnermarkt einhergeht (de Graaf/Kalmijn 2003; Fux 2011; Jaschinski 2011).

Ein weiterer zentraler Faktor für die Erklärung mütterlicher Partnerschaftsbiographien ist das Alter. Mit steigendem Alter verkleinert sich der Pool an Partnern, weil potentielle altersnahe Partner bereits gebunden sind und die physische Attraktivität abnimmt, was die eigene Position auf dem Partnermarkt verschlechtert und sich negativ auf die Partnerwahlprozesse nach einer Trennung auswirkt (Klein 2000). Da bei jüngeren Personen die

6 Einschränkend wird darauf hingewiesen, dass sich die Marital Search Theorie auf die Suche nach einem Heiratspartner bezieht und Kohabitationen nicht inkludiert. 
Neigung zur „Wiederverpartnerung“ am höchsten ist und mit steigendem Alter die Chancen für die Aufnahme einer nachehelichen Partnerschaft sinken (Bumpass et al. 1990; Klein 1990; Ermisch 2002; Wu/Schimmele 2005), erleben Kinder mit jüngeren Müttern eher multiple Übergänge: nach einer Trennung haben ihre Mütter eine höhere Wahrscheinlichkeit, einen neuen Partner zu finden. Dieser negative Alterseffekt zeigt sich für Mütter stärker als für Väter (Jaschinski 2011), was auf partnermarktspezifische Ungleichgewichte (Verengung des Partnermarkts zuungunsten älterer Frauen) oder auch auf ein bewusst vermindertes Paarbildungsverhalten von Frauen mit steigendem Alter hinweisen könnte. Für die Stabilität von Folgebeziehungen scheint hingegen das Alter eine positive Rolle zu spielen: Je älter eine Frau bei der Stieffamiliengründung, desto niedriger ist ihr Trennungsrisiko (Martin et al. 2011).

Zahlreiche Studien untersuchen den Einfluss von Kindern auf das Partnerschaftsverhalten und auf die Gründung von Partnerschaften nach einer Trennung (Lampard/ Peggs 1999; Poortman 2007; Jaschinski 2011; Lois/Kopp 2011). Überwiegend wird Kindern ein negativer Einfluss auf die Wahrscheinlichkeit, eine erneute Partnerbeziehung einzugehen, zugeschrieben. Die Verantwortung für ein Kind reduziert die Zeit und Energie, welche in das Eingehen bzw. Aufrechterhalten einer Partnerbeziehung investiert werden kann. Entsprechend sinkt mit steigender Kinderzahl die Wahrscheinlichkeit für das Eingehen neuer Partnerbeziehungen (Lampard/Peggs 1999; de Graaf/Kalmijn 2003; Lois/ Kopp 2011) bzw. werden diese später gegründet (Ermisch 2002). Tendenziell gehen Personen mit Kindern eher Partnerbeziehungen mit anderen Personen mit Kindern ein, und der negative Effekt von Kindern auf die Wahrscheinlichkeit, nach einer Trennung eine Partnerbeziehung einzugehen, ist für Mütter stärker als für Väter (Goldscheider/Sassler 2006). Der Einfluss von Kindern variiert darüber hinaus mit ihrem Alter: Jüngere Kinder stellen intensive Betreuungsanforderungen an ihre Eltern, daher bilden sie eine größere Barriere für das nacheheliche Paarbildungsverhalten (Sweeney 1997; 2010). Entsprechend zeigt Jaschinski (2011), dass geschiedene Eltern mit Kindern unter zehn Jahren eine geringere Wahrscheinlichkeit haben, eine neue Partnerbeziehung einzugehen als solche mit Kindern im Jugendalter.

Des Weiteren sind in der Erklärung mütterlicher Partnerschaftsverläufe nach einer Trennung auch zeitliche Aspekte mitbestimmend. Dies bezieht sich einerseits auf die Zeit, welche seit einer Trennung vergangen ist: So verweisen bisherige Befunde darauf, dass die Übergangswahrscheinlichkeit in eine neue Partnerschaft in den ersten zwei bis fünf Jahren nach einer elterlichen Trennung besonders hoch ist (Lankuttis/Blossfeld 2003; Jaschinski 2011). Andererseits ist auch das Scheidungs- bzw. Trennungsjahr zu berücksichtigen. In den vergangenen Jahrzehnten wurden Familienbiographien und Kindheitsverläufe insgesamt deutlich bewegter und sind von einer größeren Vielfalt geprägt, was $\mathrm{zu}$ einem Gutteil mit elterlichen Trennungen und neuerlicher (Stief-)Familiengründung zusammenhängt (Lankuttis/Blossfeld 2003; Prskawetz et al. 2003; Vikat et al. 2004; Alt/ Lange 2011). Kinder, deren Eltern sich in späteren Jahren getrennt haben, dürften demnach eine höhere Wahrscheinlichkeit für das Erleben mehrerer Übergänge haben.

Darüber hinaus wird der Partnerwahlprozess durch strukturbedingte Mechanismen eingeschränkt (Blau 1994; Klein 2001): erfolgreiche Partnerwahl erfordert adäquate Gelegenheitsstrukturen auf dem lokalen Partnerschaftsmarkt (meeting-and-mating-These). Diese können sich über das Eingebundensein in Freizeitaktivitäten, Freundschafts- oder Nachbarschaftsnetzwerke ebenso ergeben wie im Arbeits- und Berufsumfeld, welches be- 
sonders für Alleinerziehende wichtige Opportunitätsstrukturen bietet, da ihre zeitlichen Ressourcen zur Pflege sozialer Kontakte häufig limitiert sind.

\section{Datenbasis und Methoden}

Die empirischen Auswertungen basieren auf zwei österreichischen Datensätzen: dem Fertility and Family Survey (FFS) und dem Generations and Gender Survey (GGS), welche als repräsentative Umfragen unter Koordination der United Nations Economic Commission for Europe durchgeführt wurden. Aufgrund der andernfalls zu geringen Fallzahlen wurden beide Datensätze für die vorliegende Studie gepoolt. ${ }^{7} 24$ Länder partizipierten an den Fertility and Family Surveys. In Österreich fand die Datenerhebung 1995/96 mittels Computer Assisted Personal Interviews (CAPI) statt. Der Datensatz umfasst 6.120 Personen (4.581 Frauen und 1.539 Männer) im Alter von 20 bis 54 Jahren, die Rücklaufquote beträgt $72 \%$. Die Generations and Gender Surveys wurden als Nachfolgeprojekt der Fertility and Family Survey konzipiert, bisher wurden in 18 Ländern Befragungen durchgeführt. In Österreich wurden in den Jahren 2008/09 5.000 Personen (3.001 Frauen und 1.999 Männer) im Alter von 18 bis 45 Jahren mittels CAPI befragt, die Rücklaufquote beträgt $61 \%$. Obwohl die beiden Befragungen 13 Jahre auseinander liegen, erscheint eine Zusammenführung der Datensätze relativ unproblematisch, da die hier im Mittelpunkt stehenden Partnerschaftsbiographien retrospektiv erhoben wurden und sich somit überlappen. ${ }^{8}$ Berücksichtigt werden muss, dass retrospektive Datenerhebung mit einem Recall-Bias aufgrund von Erinnerungsfehlern behaftet sein kann, besonders wenn die Ereignisse schon weiter zurück liegen.

Wir beziehen uns auf Kinder, die bis zum 18. Geburtstag eine Trennung ihrer kohabitierenden Eltern erlebten ${ }^{9}$. Das mittlere Alter der Kinder im Haushalt zum Zeitpunkt der Trennung beträgt 5 Jahre und 6 Monate (Standardabweichung: 4 Jahre 4 Monate). Grundlage der Analyse sind die in beiden Datensätzen verfügbaren Partnerschaftsbiographien. Für sämtliche Beziehungen bis zum Zeitpunkt des Interviews liegen Informationen zu Monat und Jahr des Zusammenziehens, der Heirat, des Auszugs und der Scheidung vor. Für unsere Analysen wird als Beziehungsbeginn das Datum des Zusammenziehens bzw. der Heirat herangezogen, und zwar jeweils das frühere Ereignis (zumeist Kohabitation). Wir betrachten ausschließlich Partnerbeziehungen kohabitierender Partner. Living-ApartTogether-Beziehungen sind aufgrund der Limitierung durch die Daten nicht inkludiert. Als Beziehungsende gilt der Zeitpunkt des Auszugs, d.h. die räumliche Trennung. Methodisch erfordert die Analyse aus Kinderperspektive, dass jedes von den Befragten genannte Kind in den Auswertungen berücksichtigt wird.

7 Diese Vorgangsweise wurde auch in vergleichbaren Studien gewählt (Bastin 2012).

8 In einer vorbereitenden Analyse wurde in den multivariaten Modellen für die Variable Datensatz (GGS/FFS) kontrolliert; diese Variable erwies sich als nicht signifikant.

9 In die Analysen werden nur Kinder inkludiert, die zum Zeitpunkt der Trennung mit den Eltern im gemeinsamen Haushalt lebten. Zehn Kinder, die bereits ausgezogen waren, wurden aus den Analysen ausgeschlossen. 
Als Referenzbeziehung - d.h. jene Beziehung, für welche die mütterliche Partnerschaftsentwicklung nach einer Trennung betrachtet wird - gilt jeweils jene Beziehung, in die das Kind geboren wurde. Daher werden nur Partnerschaften, aus denen Kinder hervorgehen, und nur Kinder, die innerhalb einer (später getrennten) Beziehung geboren wurden, einbezogen. Kinder, die außerhalb von Beziehungen geboren wurden bzw. deren Eltern zum Zeitpunkt der Geburt nicht zusammenlebten, können nicht berücksichtigt werden, da die Dauer bis zum Eingehen einer neuen Beziehung der Mutter nicht abgeschätzt werden kann. Der entsprechende Anteil betrug 11\% unter den Geburten zwischen 1980 und 2000.

Der Datensatz lässt leider keine Schlüsse darüber zu, bei wem ein Kind in den Jahren nach der Trennung lebte. Bisherige Befunde zeigen, dass dies vorwiegend der Haushalt der Mutter ist (Figdor et al. 2006). Daher ergeben sich für Kinder zumeist weitreichende Konsequenzen aus der Partnerschaftsbiographie der Mutter, auch wenn zweifellos Übergänge in der väterlichen Beziehungsbiographie ebenfalls relevant sind. Unsere empirischen Analysen beschränken sich auf mütterliche Partnerschaftsbiographien, d.h. es werden ausschließlich Kinder von weiblichen Befragten inkludiert.

Als Übergänge werden Veränderungen in der mütterlichen Beziehungsbiographie nach der Trennung der Referenzbeziehung definiert, nämlich Heirat bzw. Zusammenziehen sowie Scheidung bzw. Trennung ${ }^{10}$. Die Konzentration auf Partnerbeziehungen der Mutter erfolgt, um eine Vermischung unterschiedlicher Variablen und daraus resultierende unspezifische Ergebnisse zu vermeiden. Zweifellos gibt es eine Reihe weiterer Übergänge, welche für Kinder nach einer elterlichen Trennung relevant sind (z.B. Hinzukommen von Halb- bzw. Stiefgeschwistern, Umzug, Schulwechsel), die in unserem Modell jedoch nicht inkludiert werden und sich auch nicht durchgängig in den Daten finden.

Von multiplen Transitionen sprechen wir, wenn ein Kind nach der Trennung der elterlichen Beziehung zumindest zwei weitere Übergänge erlebt. Veränderungen des Institutionalisierungsgrades werden nicht als Transition definiert. Lebt also ein Kind nach der Trennung mit der leiblichen Mutter und deren neuem Partner zusammen und schließen diese im weiteren Verlauf eine Ehe, stellt dies definitionsgemäß keine Transition dar. Aus Kindersicht lässt sich dies damit argumentieren, dass die Kohabitation den kindlichen Alltag zumeist stärker beeinflusst als der formale Institutionalisierungsgrad (Hawkins et al. 2006).

Wir nehmen eine Nachtrennungsperiode von acht Jahren in den Blick, kürzere Verläufe werden nicht berücksichtigt. Die Verwendung kürzerer und längerer Zeitspannen wurde ebenfalls geprüft, aber aus inhaltlichen und methodischen Gründen verworfen. Die Stichprobengröße beläuft sich auf 567 Kinder. Es werden Trennungen in der Periode 1980-2000 einbezogen (FFS: 1980-88, GGS: 1983-2000). Das multivariate Modell hat zum Ziel, die Determinanten der mütterlichen Partnerschaftsentwicklung nach einer Trennung auszumachen. Dabei werden drei verschiedene Kategorien der abhängigen Variablen „kohabitierende Partnerschaft der Mutter“ unterschieden:

(1) keine Partnerschaft der Mutter (48,5\%);

(2) neue Partnerschaft und keine Trennung dieser Beziehung (38,5\%);

(3) Trennung der neuen Partnerschaft oder mehrere Partnerschaften $(13,1 \%)$. Dies bezeichnen wir als multiple Übergänge.

10 Theoretisch könnte es sich bei diesen Übergängen auch um den biologischen Kindesvater und nicht um einen neuen Partner handeln. Dies lässt sich auf Basis der Daten nicht differenzieren. 
Welche Faktoren einen bestimmten Pfad wahrscheinlicher machen, wird mittels multinomialer logistischer Regression erschlossen (Agresti 2007). Dieses Modell wurde gewählt, weil es eine separate Schätzung der Determinanten multipler Transitionen zulässt. Die Gleichung hat folgende Form:

$$
\frac{\pi_{j}}{\pi_{J}}=e^{\alpha_{j}+\beta_{j} x}
$$

Die am stärksten besetzte Kategorie „keine Partnerschaft der Mutter“ $(\mathrm{J}=1)$ wird mit den beiden anderen Kategorien (j) gepaart, wodurch sich zwei Gleichungen ergeben, die simultan geschätzt werden. Ein Anstieg um eine Einheit in den unabhängigen Variablen (x) bewirkt eine Veränderung um $\beta_{\mathrm{j}}$ in den Odds, sich in Kategorie $\mathrm{j}$ im Vergleich zu Kategorie J zu befinden. Die durch das Einbeziehen von (potentiell) mehreren Kindern pro Familie entstehende Klumpung wurde mittels adjustierter Standardfehler korrigiert.

Die Wahl der unabhängigen Variablen ist insofern limitiert, als nur solche berücksichtigt werden können, deren Ausprägung im gesamten Beobachtungszeitraum von acht Jahren weitgehend konstant bleibt. Daher können einige potentiell relevante Einflussgrößen, wie etwa Einstellungen, Kontakthäufigkeit zum nicht-residenziellen Elternteil oder Vorhandensein einer LAT-Beziehung des residenziellen Elternteils nicht einbezogen werden. Wir haben uns zudem für ein sparsames Modell entschieden, da in multinomialen Modellen eine relativ große Anzahl an Koeffizienten geschätzt werden muss. Die fünf unabhängigen Variablen sind:

Anzahl der Geschwister zum Zeitpunkt der Trennung: Diese Variable unterscheidet Einzelkinder, Kinder mit einem sowie jene mit zwei und mehr Geschwistern. Sie inkludiert alle Kinder der Mutter, also Voll- und Halbgeschwister des Kindes.

Alter der Mutter zum Zeitpunkt der Trennung: Die Ausprägungen 17-27 Jahre, 28-31 Jahre und 32-46 Jahre wurden auf Basis von Terzilen gebildet.

Alter des jüngsten Kindes zum Zeitpunkt der Trennung: Die Kategorien 0-2 Jahre, 3-5 Jahre und 6-17 Jahre wurden auf Basis von Terzilen gebildet.

Höchste Bildung der Mutter zum Zeitpunkt der Umfrage: Die drei Bildungskategorien sind folgendermaßen definiert: „niedrig“ umfasst (nicht) abgeschlossene Pflichtschule, „mittel“ Lehre mit Berufsschule sowie Berufsbildende Mittlere Schulen ohne Matura (Abitur), „hoch“ umfasst höhere Schulen mit Matura, Akademien, Fachhochschulen und Universitäten.

Trennungsjahr: Hier werden die Jahre 1980-1989 und 1990-2000 unterschieden. Aufgrund der hohen Korrelation der Variablen Alter der Mutter bei Trennung und Alter des jüngsten Kindes bei Trennung trat im multivariaten Modell das Problem der Multikollinearität auf. Daher wurden zwei separate Modelle mit jeweils einer Variablen geschätzt. Im Ergebnisteil wird aufgrund der besseren Erklärungskraft jenes Modell, das die Variable Alter der Mutter bei Trennung einbezieht, vorgestellt. Die Resultate des zweiten Modells finden sich im Anhang und werden aufgrund der theoretischen Relevanz des Alters des jüngsten Kindes ebenfalls im Ergebnisteil diskutiert. 
Neben den genannten Variablen wurden auch die Variablen Referenzbeziehung ehelich oder unehelich, Anzahl der bisherigen Partnerschaften, Urbanität der Mutter im Alter 15 und Religionsbekenntnis der Mutter getestet, aber aufgrund ihrer geringen Erklärungskraft aus dem endgültigen Modell ausgeschlossen. Tabelle 1 stellt die Verteilungen der unabhängigen Variablen dar.

Tabelle 1: Verteilung der unabhängigen Variablen

\begin{tabular}{l|l|c}
\hline & & in Prozent \\
\hline Anzahl der Geschwister & 0 & 32,3 \\
& 1 & 40,7 \\
& $2+$ & 27,0 \\
\hline Alter der Mutter bei Trennung & bis 27 Jahre & 29,3 \\
& $28-31$ Jahre & 33,9 \\
\hline Alter des jüngsten Kindes bei Trennung & $32+$ Jahre & 42,3 \\
& $0-2$ & 26,5 \\
\hline Bildung der Mutter & $3-5$ & 31,2 \\
\hline Jahr der Trennung & $6-17$ & 31,6 \\
& Niedrig & 52,2 \\
& Mittel & 16,2 \\
\hline
\end{tabular}

Quelle: Fertility and Family Survey 1995/96 und Generations and Gender Survey 2008/09 (eigene Berechnungen)

Zusätzlich wird auch die Gruppe jener Kinder, die multiple Transitionen erlebt haben, analysiert und die vorhergesagte Wahrscheinlichkeit multipler Transitionen nach verschiedenen Charakteristika geschätzt. Dabei wird ermittelt, welche Faktoren diese Wahrscheinlichkeit reduzieren können.

\section{Ergebnisse}

\subsection{Deskription: Übergänge und Familienstrukturen nach einer elterlichen Trennung}

Zunächst wird dargestellt, wie viele Übergänge Kinder in den ersten acht Jahren nach einer elterlichen Trennung erleben und innerhalb welcher Zeitspanne diese auftreten. Wie aus Abbildung 1 ersichtlich, erleben bis zum Ende des achten Jahres nach der elterlichen Trennung 49\% keinen und 38\% einen weiteren Übergang (d.h. Zusammenziehen der Mutter mit einem Partner). Eine Betrachtung im Zeitverlauf zeigt, dass ein Großteil der Dynamiken für jene Kinder, die maximal einen Übergang erleben, in den ersten vier Jahren nach der elterlichen Trennung stattfindet. Der Anteil von Kindern, die nach der elterlichen Trennung keine weiteren Übergänge erleben, sinkt innerhalb von acht Jahren mar- 
kant: er beträgt am Ende des ersten Jahres nach der Trennung 83\%, am Ende des vierten Jahres 59\% und sinkt bis zum Ende des achten Jahres auf 49\%. Umgekehrt verläuft die Dynamik für Kinder, die einen weiteren Übergang erleben.

Der Anteil an Kindern, welche multiple Übergänge (d.h. zumindest ein partnerschaftlicher Zusammenzug und eine Trennung) erleben, ist zunächst sehr klein, steigt allerdings stetig an. Am Ende des achten Jahres nach der elterlichen Trennung haben 9\% der Kinder zwei und $4 \%$ drei oder mehr weitere Übergänge erlebt. Somit hat fast jedes siebte Kind mit getrennten Eltern im Verlauf von acht Jahren die Erfahrung multipler Transitionen gemacht und gehört damit, legt man die Ergebnisse bisheriger Forschungsarbeiten zugrunde, einer spezifischen Risikogruppe an.

Abbildung 1: Anzahl der Übergänge im mütterlichen Partnerschafts- bzw.

Kohabitationsstatus, welche Kinder innerhalb von acht Jahren nach der elterlichen Trennung erleben

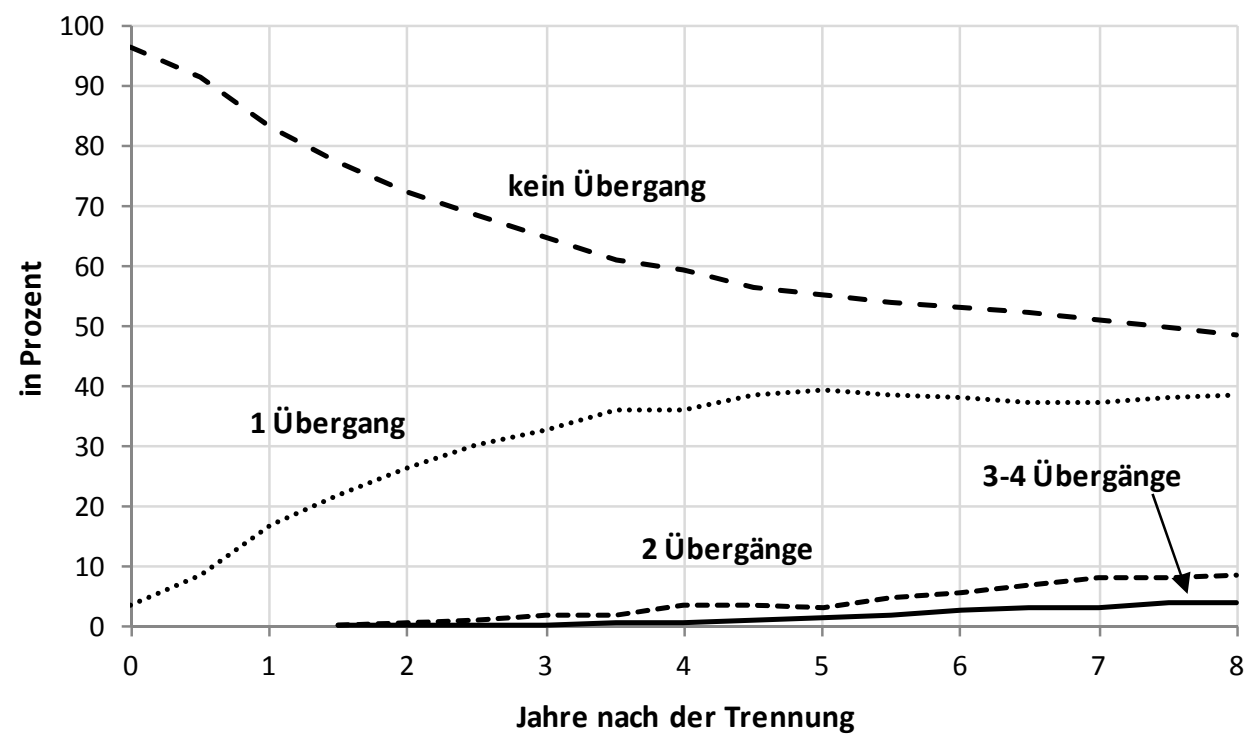

$\mathrm{n}=567$

Erläuterung: kein Übergang: keine Kohabitation der Mutter mit einem neuen Partner; 1 Übergang: Kohabitation; 2 Übergänge: eine Kohabitation und eine Trennung; 3-4 Übergänge: mehrere Kohabitationen und neuerliche Trennungen

Quelle: Fertility and Family Survey 1995/96 und Generations and Gender Survey 2008/09 (eigene Berechnungen) 
Abbildung 2: Mütterlicher Partnerschafts- bzw. Kohabitationsstatus, in welchem Kinder innerhalb von acht Jahren nach der elterlichen Trennung leben

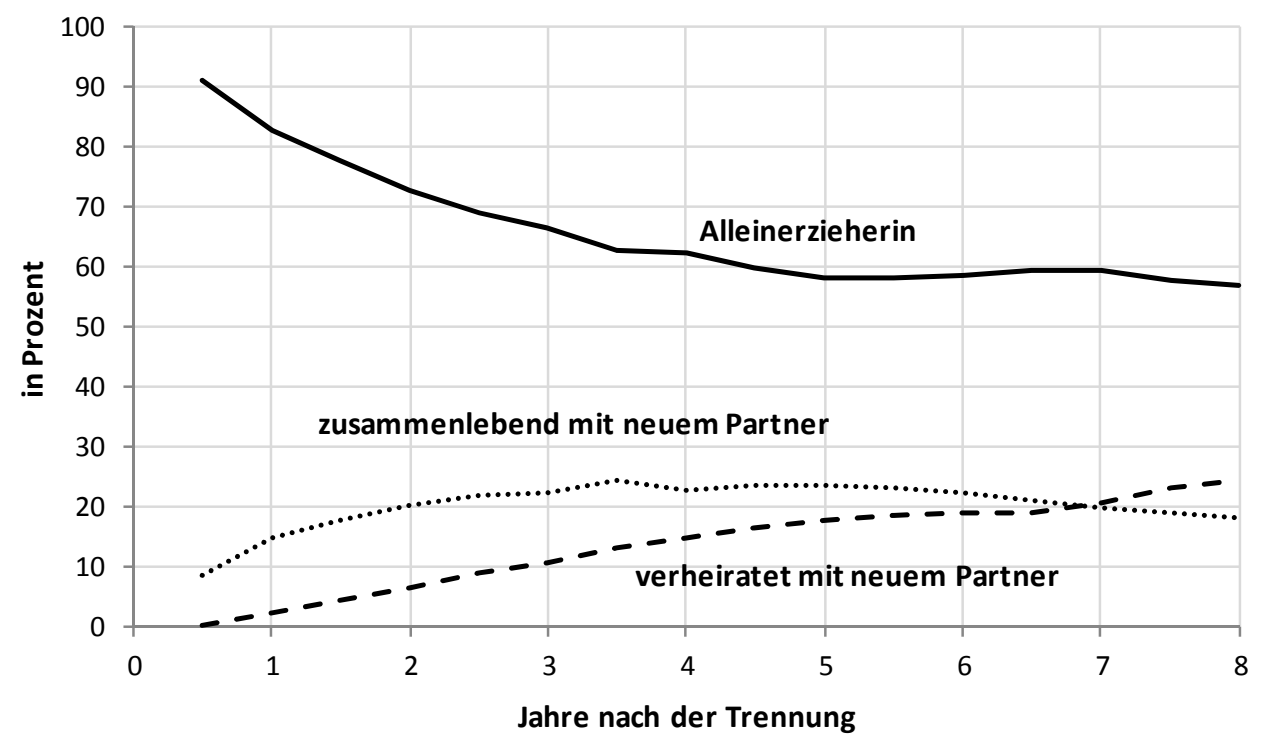

$\mathrm{n}=567$

Quelle: Fertility and Family Survey 1995/96 und Generations and Gender Survey 2008/09 (eigene Berechnungen)

Basierend auf Abbildung 2 wird nun die Frage beantwortet, in welchen familialen Lebensformen Kinder in den ersten acht Jahren nach einer elterlichen Trennung leben. Auch wenn der Fokus dieses Beitrags nicht auf dem Institutionalisierungsgrad der mütterlichen Partnerbeziehung liegt, trägt diese Analyse dazu bei, ein detaillierteres Bild der kindlichen Lebensverläufe zu erhalten. Acht Jahre nach der elterlichen Trennung leben 57\% der Kinder mit einer alleinerziehenden Mutter, d.h. ohne neuen Partner der Mutter im Haushalt. 25\% leben mit einer wiederverheirateten Mutter und 18\% mit ihrer Mutter und deren nichtehelichem Partner. Im Zeitverlauf wird ersichtlich, dass auch aus diesem Blickwinkel der Großteil der Veränderungsprozesse für Kinder, welche keine multiplen Übergänge erleben, in den ersten vier Jahren nach der Trennung stattfindet. Der Anteil jener Kinder, die mit ihrer Mutter und deren neuem Partner im Haushalt leben, steigt in dieser Zeit rasch an. Nach den ersten vier Jahren finden Veränderungen im Institutionalisierungsgrad dieser Beziehungen statt, und nichteheliche Lebensgemeinschaften werden in Ehen übergeführt. Im Detail steigen ab etwa einem halben Jahr nach der elterlichen Trennung die Anteile jener Kinder, die mit ihrer Mutter und deren neuem Partner leben. Rund $15 \%$ der Kinder werden bereits im ersten Jahr nach der elterlichen Trennung mit einer neuen kohabitierenden Partnerbeziehung der Mutter konfrontiert. Vier Jahre nach der elterlichen Trennung steigt dieser Wert auf 38\% (23\% nichtehelich, 15\% ehelich). Im Jahr Sieben nach der elterlichen Trennung ist das Verhältnis zwischen nichtehelichen und ehelichen Beziehungen schließlich ausgeglichen. 
In Bezug auf alleinerziehende Mütter wird eine auffallende Diskrepanz zwischen den Abbildungen sichtbar: $57 \%$ der Kinder leben acht Jahre nach der Trennung mit einer alleinerziehenden Mutter (Abbildung 2), aber nur 49\% der Kinder haben in diesem Zeitraum keine Übergänge erlebt (Abbildung 1). Diese Differenz ergibt sich aus einem Vergleich von Querschnitts- und Längsschnittsperspektive: Kinder, die im achten Jahr nach der Trennung mit einer alleinerziehenden Mutter leben (Querschnitt), haben aus Verlaufsperspektive entweder noch keine neue kohabitierende Partnerschaft der Mutter erlebt oder aber multiple Übergänge (nämlich entweder eine neue Kohabitation gefolgt von einer Trennung, d.h. 2 Übergänge, oder eine weitere Kohabitation sowie eine erneute Trennung, d.h. 4 Übergänge). Auf Basis der Daten zeigt sich, dass 14\% jener Kinder, die acht Jahre nach der Trennung mit einer alleinerziehenden Mutter leben, bereits multiple Übergänge erlebt haben. Dies deutet darauf hin, dass die Anzahl an Transitionen, die Kinder erleben, bei der Betrachtung aus Querschnittsperspektive tendenziell unterschätzt wird.

\subsection{Determinanten der Übergänge nach elterlicher Trennung}

Im Folgenden wird analysiert, welche Determinanten die Wahrscheinlichkeit, (multiple) Übergänge $\mathrm{zu}$ erleben, erklären können (Abbildung $3^{11}$ ). Aufgrund der besseren Lesbarkeit werden die Ergebnisse des multinomialen logistischen Modells in Form vorhergesagter Wahrscheinlichkeiten dargestellt.

Die Anzahl der Geschwister nimmt moderaten Einfluss auf die mütterlichen Partnerschaftsbiographien, die Kinder miterleben. Für Kinder mit zwei und mehr Geschwistern ist die Wahrscheinlichkeit, dass die Mutter im Verlauf von acht Jahren nach der Trennung eine Kohabitation mit einem neuen Partner eingeht, mit 23\% am geringsten; für Kinder mit keinem bzw. einem Geschwister liegt sie deutlich höher (42\%). Für die Gruppe von Kindern, welche die meisten Veränderungen erleben, nämlich eine neuerliche Trennung bzw. mehrere Partnerschaften der Mutter, besitzt die Anzahl der Geschwister keine Erklärungskraft.

Der höchste Erklärungswert kommt dem Alter der Mutter zu. Je jünger die Mutter zum Zeitpunkt der Trennung war, desto größer ist die Wahrscheinlichkeit, dass sie in den acht Jahren danach mit einem weiteren Partner lebt. Kinder, deren Mutter der jüngsten Altersgruppe angehört, weisen eine fast doppelt so hohe Wahrscheinlichkeit auf, eine neue kohabitierende Partnerbeziehung ihrer Mutter zu erleben (56\%), als Kinder mit einer Mutter in der ältesten Gruppe (32\%). Die Wahrscheinlichkeit, multiple Übergänge zu erleben, wird ebenfalls durch das Alter der Mutter erklärt: für Kinder, deren Mutter bei der Trennung bis 27 Jahre alt war, beträgt diese 20\%, während sie für Kinder mit Müttern der anderen Altersgruppen 9\% (28-31) bzw. 4\% (32 und älter) beträgt und damit signifikant niedriger liegt.

Das Alter des jüngsten Kindes beeinflusst ebenfalls, welche weitere mütterliche Partnerschaftsbiographie Kinder nach einer Trennung erleben. ${ }^{12}$ Diese Variable wird, wie erwähnt, weitgehend durch das Alter der Mutter bestimmt. Kinder in Familien, in welchen das jüngste Kind unter drei Jahre ist, haben eine höhere Wahrscheinlichkeit, eine neue

11 Das entsprechende Modell befindet sich in Tabelle A1 (Appendix).

12 Das entsprechende Modell befindet sich in Tabelle A2 (Appendix). 
mütterliche Partnerbeziehung zu erleben (51\%) als jene, in deren Familien das jüngste Kind drei Jahre oder älter ist. Auch multiple Übergänge werden eher von Kindern in Familien mit sehr jungen Kindern erlebt. Das Risiko multipler Übergänge ist signifikant höher, wenn das jüngste Kind in der Familie zwischen null und zwei ist (13\%), als wenn es sechs Jahre und älter ist (8\%).

Ein weiterer Erklärungsfaktor ist das Bildungsniveau der Mutter. Kinder, deren Mutter ein niedriges bzw. mittleres Bildungsniveau aufweist, erleben mit einer höheren Wahrscheinlichkeit (41\% bzw. 42\%) mindestens eine neue kohabitierende Partnerbeziehung ihrer Mutter als jene, deren Mutter ein hohes Bildungsniveau aufweist (32\%). Je niedriger die mütterliche Bildung, desto höher ist auch die Wahrscheinlichkeit des Kindes, multiple Übergänge zu erleben. Während diese Wahrscheinlichkeit für Kinder einer Mutter mit niedriger Bildung $11 \%$ beträgt, liegt sie für Kinder einer Mutter mit hoher Bildung bei $6 \%$. Die einzelnen Bildungsgruppen unterscheiden sich nicht signifikant, was vermutlich auf die geringen Fallzahlen zurückgeführt werden kann.

Auch das Jahr der Trennung ist eine wesentliche Determinante der Erklärung kindlicher Lebensverläufe. Während in der älteren Kohorte (1980-1989) die Wahrscheinlichkeit für mindestens eine neue kohabitierende Partnerschaft der Mutter bei $42 \%$ liegt, beträgt sie für die jüngere Kohorte (1990-2000) 45\%. Die Wahrscheinlichkeit für multiple Transitionen ist im Zeitverlauf deutlich gestiegen und ist in der jüngeren Kohorte signifikant höher (16\%) als in der älteren $(9 \%)$.

Abbildung 3: Determinanten der Anzahl der Übergänge im mütterlichen Partnerschaftsbzw. Kohabitationsstatus, welche Kinder innerhalb von acht Jahren nach der elterlichen Trennung erleben (vorhergesagte Wahrscheinlichkeiten)

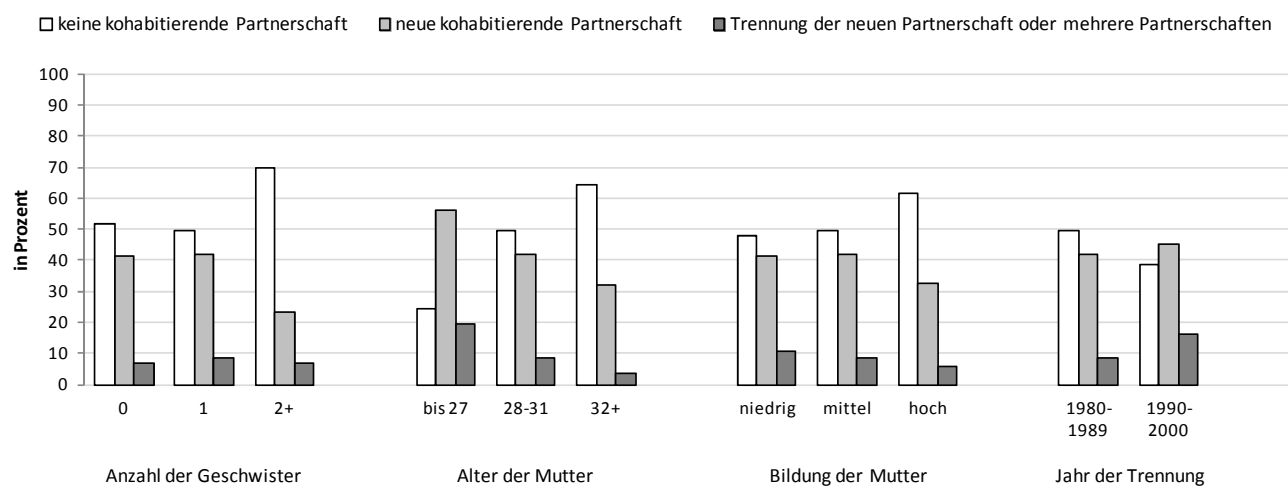

$\mathrm{n}=567$

Quelle: Fertility and Family Survey 1995/96 und Generations and Gender Survey 2008/09 (eigene Berechnungen)

Abschließend wird nun gesondert die Gruppe jener Kinder, die nach der elterlichen Trennung multiple Übergänge erleben, analysiert. Zunächst wird geschätzt, wie hoch die Wahrscheinlichkeit für ein Kind ist, multiple Transitionen zu erleben, wenn alle bisher vorgestellten Faktoren dies begünstigen. Ausgangspunkt ist ein Kind, das zwei oder mehr 
Geschwister hat. Die Mutter ist zum Zeitpunkt der Trennung 27 Jahre oder jünger, sie hat ein niedriges Bildungsniveau, und das Jahr der Trennung liegt zwischen 1990 und 2000. Treffen alle genannten Faktoren zu, so besitzt das Kind eine Wahrscheinlichkeit von 38\%, multiple Transitionen zu erleben.

Nun gehen wir der Frage nach, welche hauptsächlichen Faktoren diese hohe Wahrscheinlichkeit multipler Übergänge in der genannten Gruppe reduzieren können. Die Ergebnisse sind in Tabelle 2 dargestellt und unterstreichen nochmals die Resultate der bisherigen Analysen: Ist die Bildung der Mutter nicht niedrig, sondern hoch, verringert sich die Wahrscheinlichkeit multipler Übergänge auf 21\%. Fand die Trennung nicht zwischen 1990-2000 statt, sondern bereits in der Kohorte 1980-1989, so reduziert dies die Wahrscheinlichkeit multipler Übergänge auf 19\%. Im Zeitverlauf ergibt sich also eine Halbierung des Risikos. Einen noch deutlich stärkeren Einfluss zeigt das Alter der Mutter zum Zeitpunkt der Trennung: beträgt dieses nicht maximal 27 Jahre, sondern ist die Mutter bereits 32 Jahre oder älter, so reduziert sich die Wahrscheinlichkeit multipler Übergänge auf $7 \%$.

Tabelle 2: Vorhergesagte Wahrscheinlichkeit multipler Übergänge nach verschiedenen Charakteristika (multinomiales logistisches Modell)

\begin{tabular}{l|c}
\hline Charakteristika & Wahrscheinlichkeit für multiple Übergänge \\
\hline - 2+ Geschwister & $38,1 \%$ \\
- Alter der Mutter bei Trennung: bis 27 Jahre & \\
- Niedrige Bildung der Mutter & \\
- Trennung 1990-2000 & \\
\hline - 2+ Geschwister & $21,1 \%$ \\
- Alter der Mutter bei Trennung: bis 27 Jahre & \\
- Hohe Bildung der Mutter & \\
- Trennung 1990-2000 & \\
- 2+ Geschwister & $19,2 \%$ \\
- Alter der Mutter bei Trennung: bis 27 Jahre & \\
- Niedrige Bildung der Mutter & \\
- Trennung 1980-1989 & \\
\hline - 2+ Geschwister & \\
- Alter der Mutter bei Trennung: 32+ Jahre & \\
- Niedrige Bildung der Mutter & \\
- Trennung 1990-2000 & \\
\hline $\mathrm{n}=567$ &
\end{tabular}

Quelle: Fertility and Family Survey 1995/96 und Generations and Gender Survey 2008/09 (eigene Berechnungen)

\section{Zusammenfassung und Diskussion}

Die Zielsetzung des vorliegenden Beitrags war es, detailliert im Zeitverlauf zu analysieren, wie viele Übergänge Kinder in Österreich nach einer elterlichen Trennung erleben, wann diese stattfinden, in welchen mütterlichen Partnerschaftskonstellationen und familialen Lebensformen Kinder danach leben, und welche Determinanten die Wahrschein- 
lichkeit von Transitionen beeinflussen. Damit wurde die bestehende Forschungslücke zur Häufigkeit familialer Übergänge nach einer elterlichen Trennung geschlossen.

Wir konnten zeigen, dass für jene Kinder, die nach der elterlichen Trennung keinen oder maximal einen Übergang erleben, ein Großteil der Dynamiken in den ersten vier Jahren nach der elterlichen Trennung stattfindet. In dieser Zeit erleben Kinder am ehesten, dass ihre Mutter mit einem neuen Partner zusammenzieht. Danach werden vielfach bereits bestehende Lebensgemeinschaften in Ehen überführt, und der Anteil jener Kinder, die multiple Transitionen erleben, steigt an. Dies entspricht in etwa bisherigen Befunden (Lankuttis/Blossfeld 2003; Wu/Schimmele 2005; Jaschinski 2011). Insgesamt haben in den ersten acht Jahren nach der Trennung rund 50\% der Kinder keine und rund $40 \%$ genau eine weitere Transition erlebt. Fast jedes siebte Kind mit getrennten Eltern (13\%) macht im Verlauf von acht Jahren die Erfahrung multipler Übergänge und gehört damit einer spezifischen Risikogruppe an.

Die Analyse der Determinanten der dahinter liegenden Dynamiken zeigt, dass die Lebensverläufe von Kindern nach einer elterlichen Trennung sowie die Wahrscheinlichkeit, multiple Transitionen zu erleben, besonders durch das Alter der Mutter erklärt werden können. Je jünger die Mutter zum Zeitpunkt der Trennung war, desto wahrscheinlicher ist es, dass sie eine neue kohabitierende Partnerbeziehung eingeht. Außerdem verweist die Analyse kindbezogener Variablen darauf, dass multiple Transitionen mit deutlich höherer Wahrscheinlichkeit in Familien mit sehr jungen Kindern stattfinden. Diese Alterseffekte machen deutlich, dass früh erlebte Übergänge eng mit einer größeren Anzahl an Übergängen verwoben sind, und entsprechen bislang vorliegenden Ergebnissen (Lampard/Peggs 1999; Poortman 2007; Jaschinski 2011). Sie spiegeln einerseits die Mechanismen des Partnermarktes wider, wonach jüngere Frauen eine höhere Wahrscheinlichkeit haben, eine neue Partnerbeziehung einzugehen (Bumpass et al. 1990; Klein 1990; Ermisch 2002; $\mathrm{Wu} /$ Schimmele 2005), und sind andererseits konsistent mit Befunden, wonach ein höheres Alter der Mutter bei Stieffamiliengründung stabilere Lebensverläufe für Kinder nach einer elterlichen Trennung impliziert (Martin et al. 2011).

Darüber hinaus ist ein deutlicher Effekt des Trennungsjahres zu beobachten. Der Kohortenvergleich (1980-1989 sowie 1990-2000) zeigt, dass im Zeitverlauf die Wahrscheinlichkeit für Kinder, multiple Übergänge zu erleben, stark angestiegen ist. Aus Kindersicht bedeutet dies, dass elterliche Trennung zunehmend mit bewegten weiteren Verläufen verbunden ist. Es stellt sich die Frage, ob die steigende Häufigkeit von Transitionen zu einer „Normalisierung“ führen und so künftig die bislang bekannten negativen Auswirkungen multipler Übergänge verringern könnte. Mit den vorliegenden Daten lässt sich diese Frage nicht beantworten, und auch die fachliche Diskussion zu diesem Thema ist von sehr unterschiedlichen Befunden geprägt: Während einige Autor(inn)en eine elterliche Trennung generell als negativ für Kinder betrachten und davon ausgehen, dass es aus Kindersicht eine „gute Trennung“ nicht geben kann (Amato et al. 2011; Pryor 2011), betonen andere, dass dies unter bestimmten Rahmenbedingungen durchaus vorstellbar sei (Ahrons 2011). Qualitative Befunde für Österreich verweisen darauf, dass Scheidung und Trennung trotz gestiegener Häufigkeiten und höherer normativer Akzeptanz nach wie vor als negative, eine Desorganisation der Familie verursachende Ereignisse betrachtet werden (Zartler 2012b). Dies ließe darauf schließen, dass ein Ansteigen multipler Transitionen nicht notwendigerweise positive Konsequenzen für Kinder im Sinne einer „Normalisierung“ nach sich zieht. 
Ein weiterer Erklärungsfaktor ist das mütterliche Bildungsniveau. Je höher die mütterliche Bildung, desto niedriger ist die Wahrscheinlichkeit des Kindes, eine neue mütterliche Partnerbeziehung zu erleben. Dieses Ergebnis steht nicht im Einklang mit einigen Befunden (de Graaf/Kalmijn 2003; Fux 2011; Jaschinski 2011), kann aber damit erklärt werden, dass für hoch gebildete Frauen sowohl der Pool an geeigneten Partnern als auch der ökonomische Druck, eine neue Partnerbeziehung einzugehen, geringer ist als für jene mit niedriger Bildung (Lankuttis/Blossfeld 2003; Ott et al. 2011). Daraus resultierend ist eine Ausdifferenzierung kindlicher Lebenschancen abhängig von der Bildung ihrer Mütter denkbar (McLanahan 2004; Esping-Andersen 2009; Bertram et al. 2011).

Abschließend seien einige methodische Limitierungen des Beitrags erwähnt. Zunächst enthalten die verwendeten Datensätze keine Information über Partner, die nicht im gemeinsamen Haushalt leben. Dies führt zu einer Unterschätzung der Anzahl von neuen Beziehungen. Des Weiteren ist anzumerken, dass Trennungen, die früh im Beobachtungszeitraum stattfinden, die Wahrscheinlichkeit für multiple Transitionen erhöhen, da für diese Fälle ein größerer Beobachtungszeitraum erfasst wird, und somit nicht ausgeschlossen werden kann, dass das gewählte Modell eventuell nicht hinreichend zwischen früh erlebten ersten und zahlreichen weiteren Übergängen unterscheiden kann. Darüber hinaus wird im vorliegenden Datenmaterial angenommen, dass die Kinder hauptsächlich bei ihrer Mutter leben. Eine Differenzierung nach residenziellen und nicht-residenziellen Elternteilen würde die Analyse zusätzlich bereichern. Das Forschungspotenzial in Hinblick auf haushaltsübergreifende Familienkonstellationen erscheint groß, und zwar sowohl hinsichtlich quantitativer als auch qualitativer Aspekte (Entfernung, Zeit mit dem jeweiligen Elternteil, Beziehungsqualität).

Welche Implikationen ergeben sich für künftige Forschung in diesem Themenbereich? Sowohl für theoretische als auch empirische Zugänge erscheint es zielführend, starkes Augenmerk auf die Bedeutung familialer Übergänge zu legen und dieser Perspektive gegenüber einer statischen familienstrukturellen Betrachtung den Vorzug zu geben (Amato 2010). Unbedingt zu berücksichtigen ist dabei die Komplexität von Transitionsprozessen (Anderson/Greene 2005). Um ihre Bedeutung für Kinder, aber auch für Eltern und andere involvierte Personen angemessen erfassen zu können, sind Primärerhebungen unabdingbar. Ein Methodenmix aus quantitativen und qualitativen Zugängen scheint hier vielversprechend (Saint-Jacques et al. 2011). Die Inklusion von Beziehungen mit Partnern außerhalb des Haushalts und die Beschäftigung mit der Frage, welche Übergänge Kinder diesbezüglich erleben, ist ebenso erforderlich wie eine Ausweitung der Analysen auf den nicht-residenziellen Elternteil. Zudem sollten die Abläufe, Determinanten und Auswirkungen unterschiedlicher Transitionen verstärkt untersucht werden. Dabei geht es auch um die Frage, ob verschiedene Übergänge (Hinzukommen eines neuen Partners, neuerliche Trennung, usw.) jeweils als ähnlich stressreich für Kinder betrachtet werden können, und wie sich die Kumulation unterschiedlicher Übergänge auswirkt. Die Verknüpfung der hier vorliegenden Ergebnisse mit weiteren Variablen wie kindlichen Entwicklungsparametern oder dem Konfliktniveau von Familien würde ebenfalls eine Bereicherung darstellen (Wendt/Walper 2007; Schneewind/Walper 2008). Jedenfalls erscheint aufgrund der im Zeitverlauf gestiegenen Wahrscheinlichkeit für Übergänge und der daraus resultierenden Variabilität von Verläufen nach der elterlichen Trennung eine weitere Bearbeitung und Ausdifferenzierung der Perspektive multipler Transitionen zukunftsweisend. 


\section{Literatur}

Acock, A. C. \& Demo, D. H. (1994). Family diversity and well-being. Thousand Oaks: Sage.

Agresti, A. (2007). An introduction to categorical data analysis. New Jersey: John Wiley.

Ahrons, C. R. (2011). Commentary on "Reconsidering the 'good divorce"'. Family Relations, 60, 5, S. 528-532.

Alt, C. \& Bender, D. (1998). Kinder in nichtehelichen Lebensgemeinschaften. In: Bien, W. \& Schneider, N. F. (Hrsg.), Kind ja, Ehe nein? Status und Wandel der Lebensverhältnisse von nichtehelichen Kindern und Kindern in nichtehelichen Lebensgemeinschaften. Opladen: Leske + Budrich (DJI: Familiensurvey 7), S. 141-173.

Alt, C. \& Lange, A. (2011). Kindschaftskonstellationen in Vater-Mutter-Familien und in Einelternfamilien. In: Schwab, D. \& Vaskovics, L. A. (Hrsg.), Pluralisierung der Elternschaft und Kindschaft. Familienrecht, -soziologie und -psychologie im Dialog. Opladen \& Farmington Hills, MI: Verlag Barbara Budrich (Sonderheft 8 der Zeitschrift für Familienforschung/Journal of Family Research), S. 139-156.

Amato, P. R. (1993). Children's adjustment to divorce: Theories, hypotheses, and empirical support. Journal of Marriage and the Family, 55, 1, S. 23-38.

Amato, P. R. (2000). The consequences of divorce for adults and children. Journal of Marriage and Family, 62, 4, S. 1269-1287.

Amato, P. R. (2010). Research on divorce: Continuing trends and new developments. Journal of Marriage and Family, 72, 3, S. 650-666.

Amato, P. R., Kane, J. B. \& James, S. (2011). Reconsidering the "good divorce". Family Relations, 60, 5, S. 511-524.

Amato, P. R. \& Sobolewski, J. M. (2001). The effects of divorce and marital conflict on adults children's psychological well-being. American Sociological Review, 66, 6, S. 900-921.

Anderson, E. R. \& Greene, S. M. (2005). Transitions in parental repartnering after divorce. Journal of Divorce and Remarriage, 43, 3, S. 47-62.

Aquilino, W. S. (1996). The life course of children born to unmarried mothers: Childhood living arrangements and young adult outcomes. Journal of Marriage and Family, 58, 2, S. 293-210.

Bastin, S. (2012). Dynamik alleinerziehender Mutterschaft. Partnerschaftsverläufe in der frühen Elternbiographie. In: Huinink, J., Kreyenfeld,, M. \& Trappe, H. (Hrsg.), Familie und Partnerschaft in Ostund Westdeutschland. Ähnlich und doch immer noch anders. Opladen, Berlin \& Toronto: Verlag Barbara Budrich (Sonderheft 9 der Zeitschrift für Familienforschung/Journal of Family Research), S. 201-228.

Bertram, H., Kohl, S. \& Rösler, W. (2011). Zur Lage der Kinder in Deutschland 2011/2012. Kindliches Wohlbefinden und gesellschaftliche Teilhabe. Köln: Deutsches Komitee für UNICEF.

Blau, P. M. (1994). Structural contexts of opportunities. Chicago: University of Chicago Press.

Bumpass, L., Sweet, J. \& Castro Martin, T. (1990). Changing patterns of remarriage. Journal of Marriage and Family, 52, 3, S. 747-756.

Cavanagh, S. E. \& Huston, A. C. (2006). Family instability and children's early problem behaviour. Social Forces, 85, 1, S. 551-585.

Cooper, C., McLanahan, S., Meadows, S. \& Brooks-Gunn, J. (2009). Family structure transitions and maternal parenting stress. Journal of Marriage and Family, 71, 3, S. 558-574.

Cowan, P. A. \& Hetherington, E. M. (1991). Family transitions. Advances in family research. Hillsdale: Erlbaum.

de Graaf, P. \& Kalmijn, M. (2003). Alternative routes in the remarriage market: competing-risk analyses of union formation after divorce. Social Forces, 81, 4, S. 1459-1498.

Edin, K. \& Kefalas, M. (2005). Promises I can keep. Why poor women put motherhood before marriage. Berkeley: University of California Press.

Ermisch, J. (2002). Trying again: Repartnering after dissolution of a union. University of Essex: Institute for Social and Economic Research (Working Paper 2002-19). 
Esping-Andersen, G. (1990). The three worlds of welfare capitalism. Cambridge: Polity Press.

Esping-Andersen, G. (2009). The incomplete revolution: Adapting to women's new roles. Cambridge: Polity Press.

Feldhaus, M., Boehnke, M. \& Krohn, F. (2011). Intergenerationale Transmission von Familienverläufen: Vererben sich familiale Verläufe? Vortrag bei der 2. Nutzerkonferenz des Beziehungs- und Familienpanels pairfam, 23. Juni 2011, Technische Universität Chemnitz.

Feldhaus, M. \& Huinink, J. (2011). Multiple Elternschaften in Deutschland - eine Analyse zur Vielfalt von Elternschaft in Folgepartnerschaften. In: Schwab, S. \& Vaskovics, L. A. (Hrsg.), Pluralisierung der Elternschaft und Kindschaft. Familienrecht, -soziologie und -psychologie im Dialog. Opladen \& Farmington Hills, MI: Verlag Barbara Budrich (Sonderheft 8 der Zeitschrift für Familienforschung/Journal of Family Research), S. 77-104.

Figdor, H., Barth-Richartz, J., Kränzl-Nagl, R. \& Pelikan, C. (2006). Evaluationsstudie über die Auswirkungen der Neuregelungen des KindR $\ddot{A} G$ 2001, insbesondere der Obsorge beider Eltern. Wien: Bundesministerium für Justiz.

Flowerdew, J. \& Neale, B. (2003). Trying to stay apace: Children with multiple challenges in their postdivorce family lives. Childhood, 10, 2, S. 147-162.

Fomby, P. \& Cherlin, A. J. (2007). Family instability and child well-being. American Sociological Review, 72, S. 181-204.

Fux, B. (2011). Sozioökonomische Situation und soziale Beziehungen von Alleinerziehenden. Würzburg: Ergon.

Goldscheider, F. \& Sassler, S. (2006). Creating stepfamilies: Integrating children into the study of union formation. Journal of Marriage and Family, 68, 2, S. 275-291.

Graefe, D. R. \& Lichter, D. T. (2007). When unwed mothers marry. The marital and cohabiting partners of midlife women. Journal of Family Issues, 28, 5, S. 595-622.

Haas, B. (2009). Geschlechtergerechte Arbeitsteilung - theoretisch ja, praktisch nein! Eine Bilanz für österreichische Paarhaushalte mit Kindern. In: E. Appelt (Hrsg.), Gleichstellungspolitik in Österreich. Eine kritische Bilanz. Innsbruck, Wien \& Bozen: Studienverlag, S. 135-148.

Hawkins, D. N., Amato, P. R. \& King, V. (2006). Parent-adolescent involvement: The relative influence of parent gender and residence. Journal of Marriage and Family, 68, 1, S. 125-136.

Hill, M. S., Yeung, W.-J. J. \& Duncan, G. J. (2001). Childhood family structure and young adult behaviors. Journal of Population Economics, 14, 2, S. 271-299.

Hopf, G. (2011). Reformen und Reformbedarf des österreichischen Familienrechts vor dem Hintergrund europäischer Entwicklungen. In: Borić, T., Lurger, B., Schwarzenegger, P. \& Terlitza, U. (Hrsg.), Öffnung und Wandel - Die internationale Dimension des Rechts. Wien: Lexis Nexis, S. 241-256.

Jaschinski, I. (2011). Der Übergang in eine nacheheliche Partnerschaft: Eine vergleichende Analyse zwischen Männern und Frauen auf Basis des deutschen Generations and Gender Survey. Zeitschrift für Familienforschung/Journal of Family Research, 23, 2, S. 219-240.

Jensen, A.-M. (2009). Mobile children: Small captives of large structures? Children \& Society, 23, 2, S. 123-135.

Juby, H., Billette, J.-M., Laplante, B. \& Le Bourdais, C. (2007). Nonresident fathers and children: Parents' new unions and frequency of contact. Journal of Family Issues, 28, 9, S. 1220-1245.

Kiernan, K., McLanahan, S., Holmes, J. \& Wright, M. (2011). Fragile families in the US and UK. Princeton: Center for Research on Child Well-Being (Working Paper WP 11-04-FF).

Kiernan, K. \& Mensah, F. (2010). Partnership trajectories, parent and child well-being. In: Hansen, K., Joshi, H. \& Dex, S. (Hrsg.), Children of the 21st century. The first five years. Bristol: Policy Press, S. 77-94.

Klein, T. (1990). Wiederheirat nach Scheidung in der Bundesrepublik. Eine empirische Überprüfung bislang vorliegender Theoreansätze aus der Perspektive des Lebensverlaufs. Kölner Zeitschrift für Soziologie und Sozialpsychologie, 42, 1, S. 60-80.

Klein, T. (2000). Partnerwahl zwischen sozialstrukturellen Vorgaben und individueller Entscheidungsautonomie. Zeitschrift für Soziologie der Erziehung und Sozialisation, 20, S. 229-243. 
Klein, T. (2001) (Hrsg.). Partnerwahl und Heiratsmuster. Sozialstrukturelle Voraussetzungen der Liebe. Opladen: Leske + Budrich.

Lampard, R. \& Peggs, K. (1999). Repartnering: The relevance of parenthood and gender to cohabitation and remarriage among the formerly married. British Journal of Sociology, 50, 3, S. 443-465.

Lankuttis, T. \& Blossfeld, H.-P. (2003). Determinanten der Wiederheirat nach der ersten Scheidung in der Bundesrepublik Deutschland. Zeitschrift für Familienforschung, 15, 1, S. 5-24.

Lenz, K. (2006). Soziologie der Zweierbeziehung. Eine Einführung. Wiesbaden: VS Verlag für Sozialwissenschaften.

Lois, D. \& Kopp, J. (2011). Elternschaftskonstellationen bei Alleinerziehenden. In: Schwab, D. \& Vaskovics, L. A. (Hrsg.), Pluralisierung der Elternschaft und Kindschaft. Familienrecht, -soziologie und -psychologie im Dialog. Opladen \& Farmington Hills, MI: Verlag Barbara Budrich (Sonderheft 8 der Zeitschrift für Familienforschung/Journal of Family Research), S. 59-76.

Martin, V., Le Bourdais, C. \& Lapierre-Adamcyk, É. (2011). Stepfamily instability in Canada - The impact of family composition and union type. Zeitschrift für Familienforschung/Journal of Family Research, 23, 2, S. 196-218.

McLanahan, S. (2004). Diverging destinies: How children are faring under the second demographic transition. Demography, 41, 4, S. 607-627.

Oppenheimer, V. K. (1988). A theory of marriage timing. American Journal of Sociology, 94, 3, S. 563-591.

Osborne, C. \& McLanahan, S. (2007). Partnership instability and child well-being. Journal of Marriage and Family, 69, 4, S. 1065-1083.

Ott, N., Hancioglu, M. \& Hartmann, B. (2011). Dynamik der Familienform „alleinerziehend“. Gutachten. Berlin: Bundesministerium für Arbeit und Soziales.

Pfau-Effinger, B. \& Lewis, J. (1995). Gender and the evolution of European social policies. In: Leibfried, S. \& Pierson, P. (Hrsg.), European social policy. Between fragmentation and integration. Washington: Brookings, S. 4-64.

Poortman, A.-R. (2007). The first cut is the deepest? The role of the relationship career for union formation. European Sociological Review, 23, 5, S. 585-598.

Prskawetz, A., Vikat, A., Philipov, D. \& Engelhardt, H. (2003). Pathways to stepfamily formation in Europe: Results from the FFS. Demographic Research, 8, 5, S. 107-150.

Pryor, J. (2011). Commentary on "Reconsidering the 'good divorce"'. Family Relations, 60, 5, S. 525527.

Rutter, M. (1981). Stress, coping and development: some issues and some questions. Journal of Child Psychology and Psychiatry, 22, 4, S. 323-356.

Saint-Jacques, M.-C., Robitaille, C., Godbout, É., Parent, C., Drapeau, S. \& Gagne, M.-H. (2011). The processes distinguishing stable from unstable stepfamily couples: a qualitative analysis. Family Relations, 60, S. 545-561.

Schneewind, K. A. \& Walper, S. (2008). Kinder in verschiedenen Familienformen. In: Hasselhorn, M. \& Silbereisen, R. K. (Hrsg.), Entwicklungspsychologie des Säuglings- und Kindesalters. Göttingen: Hogrefe, S. 571-616.

Statistik Austria (Hrsg.) (2011). Demographische Indikatoren 2010. Wien: Statistik Austria.

Statistik Austria (Hrsg.) (2012). Demographisches Jahrbuch 2011. Wien: Verlag Österreich.

Statistik Austria (2013a). Ehescheidungen seit 2012 nach ausgewählten Merkmalen. http://www. statistik.at/web_de/statistiken/bevoelkerung/scheidungen/022912.htm (Stand: 2013-08-03).

Statistik Austria (Hrsg.) (2013b). Familien- und Haushaltsstatistik 2012. Wien: Verlag Österreich.

Stegmann, D. (1997). Lebensverläufe Alleinerziehender in West- und Ostdeutschland. Wiesbaden: Bundesinstitut für Bevölkerungsforschung.

Sun, Y. \& Li, Y. (2008). Stable postdivorce familiy structures during late adolescence and socioeconomic consequences in adulthood. Journal of Marriage and Family, 70, 1, S. 129-143.

Sweeney, M. M. (1997). Remarriage of women and men after divorce: The role of socioeconomic prospects. Journal of Family Issues, 18, 5, S. 479-502.

Sweeney, M. M. (2010). Remarriage and stepfamilies: Strategic sites for family scholarship in the 21 st century. Journal of Marriage and Family, 72, 3, S. 667-684. 
Tach, L., Mincy, R. B. \& Edin, K. (2010). Parenting as a 'package deal': Relationships, fertility, and nonresident father involvement among unmarried parents. Demography, 47, 1, S. 181-204.

Vikat, A., Thomson, E. \& Prskawetz, A. (2004). Childrearing responsibility and stepfamily fertility in Finland and Austria. European Journal of Population, 20, 1, S. 1-21.

Waldfogel, J., Craigie, T.-A. \& Brooks-Gunn, J. (2010). Fragile families and child wellbeing. The Future of Children, 20, 2, S. 87-112.

Wendt, E.-V. \& Walper, S. (2007). Entwicklungsverläufe von Kindern in Ein-Eltern- und Stieffamilien. In: Alt, C. (Hrsg.), Kinderleben - Start in die Grundschule. Band 3: Ergebnisse aus der zweiten Welle. Wiesbaden: VS Verlag für Sozialwissenschaften, S. 211-242.

Wu, Z. \& Schimmele, C. M. (2005). Repartnering after first union disruption. Journal of Marriage and Family, 67, 1, S. 27-36.

Zartler, U. (2012a). Das Familienbild des ABGB und die Lebenssituation von Scheidungs- und Nachscheidungsfamilien. Beiträge zur Rechtsgeschichte Österreichs, 2, 1, S. 44-56.

Zartler, U. (2012b). Die Kernfamilie als Ideal. Zur Konstruktion von Scheidung und Nachscheidungsfamilien. Zeitschrift für Familienforschung/Journal of Family Research, 24, 1, S. 67-84.

Zartler, U. (2013). Nichteheliche Lebensgemeinschaften. Soziologische Analyse einer Lebensform. In: Österreichischer Juristentag (Hrsg.), Verhandlungen des Achtzehnten Österreichischen Juristentages, Linz 2012. Zivilrecht: Neue Regelungen für nichteheliche Lebensgemeinschaften? Wien: Manz, S. 24-46.

Zartler, U. \& Beham, M. (2011). Alleinerziehen. Alltägliche Herausforderungen im Umgang mit knappen Ressourcen. SWS-Rundschau, 51, 4, S. 383-404.

Eingereicht am/Submitted on: 21.11.2012

Angenommen am/Accepted on: 30.04.2013

Anschriften der Autorinnen/Addresses of the authors:

Dr. Ulrike Zartler (Korrespondenzautorin/Corresponding author)

Universität Wien

Institut für Soziologie

Rooseveltplatz 2

1090 Wien

Österreich/Austria

Dr. Caroline Berghammer

Universität Wien

Institut für Soziologie

Rooseveltplatz 2

$1090 \mathrm{Wien}$

Österreich/Austria

Wittgenstein Centre for Demography and Global Human Capital (IIASA, VID/ÖAW, WU)

Vienna Institute of Demography/Austrian Academy of Sciences

Wohllebengasse 12-14/6. Stock

$1040 \mathrm{Wien}$

Österreich/Austria

E-Mail: ulrike.zartler@univie.ac.at

E-Mail: caroline.berghammer@univie.ac.at 


\section{Appendix}

Tabelle A1: Determinanten der Anzahl der Übergänge im mütterlichen Partnerschaftsbzw. Kohabitationsstatus, welche Kinder innerhalb von acht Jahren nach der elterlichen Trennung erleben (multinomiales logistisches Modell)

\begin{tabular}{|c|c|c|c|}
\hline $\begin{array}{l}\text { Ref.: Keine kohabitierende } \\
\text { Partnerschaft der Mutter }\end{array}$ & $\begin{array}{c}\text { Neue kohabitierende } \\
\text { Partnerschaft }\end{array}$ & $\begin{array}{l}\text { Trennung der Partnerschaft } \\
\text { oder mehrere Partnerschaften }\end{array}$ & \\
\hline 1 & 2 & 3 & $\mathrm{n}$ \\
\hline \multicolumn{4}{|l|}{ Anzahl der Geschwister } \\
\hline 0 & 0,95 & 0,73 & 183 \\
\hline 1 (ref.) & 1 & 1 & 231 \\
\hline $2+$ & $0,39^{* *}$ & 0,56 & 153 \\
\hline \multicolumn{4}{|l|}{ Alter der Mutter } \\
\hline Bis 27 & $2,73^{\star \star *}$ & $4,55^{\star \star *}$ & 206 \\
\hline 28-31 (ref.) & 1 & 1 & 169 \\
\hline $32+$ & 0,59 & $0,33^{*}$ & 192 \\
\hline \multicolumn{4}{|l|}{ Bildung der Mutter } \\
\hline Niedrig & 1,01 & 1,30 & 179 \\
\hline Mittel (ref.) & 1 & 1 & 296 \\
\hline Hoch & 0,62 & 0,54 & 92 \\
\hline \multicolumn{4}{|l|}{ Jahr der Trennung } \\
\hline 1980-1989 (ref.) & 1 & 1 & 342 \\
\hline $1990-2000$ & 1,38 & $2,41^{* *}$ & 225 \\
\hline Pseudo R2 & 0,11 & & \\
\hline N & 567 & & \\
\hline Df & 14 & & \\
\hline
\end{tabular}

Signifikanzniveaus: $* \mathrm{p}<0,10, * * \mathrm{p}<0,05, * * * \mathrm{p}<0,01$

Quelle: Fertility and Family Survey 1995/96 und Generations and Gender Survey 2008/09 (eigene Berechnungen) 
Tabelle A2: Determinanten der Anzahl der Übergänge im mütterlichen Partnerschafts- bzw. Kohabitationsstatus, welche Kinder innerhalb von acht Jahren nach der elterlichen Trennung erleben ${ }^{\text {a }}$ (multinomiales logistisches Modell)

\begin{tabular}{|c|c|c|c|}
\hline $\begin{array}{l}\text { Ref.: Keine kohabitierende } \\
\text { Partnerschaft der Mutter } \\
1\end{array}$ & $\begin{array}{c}\text { Neue kohabitierende } \\
\text { Partnerschaft } \\
2\end{array}$ & $\begin{array}{c}\text { Trennung der Partnerschaft } \\
\text { oder mehrere Partnerschaften } \\
3\end{array}$ & $\mathrm{n}$ \\
\hline \multicolumn{4}{|l|}{ Anzahl der Geschwister } \\
\hline 0 & 1,33 & 1,29 & 183 \\
\hline 1 (ref.) & 1 & 1 & 231 \\
\hline $2+$ & $0,33^{* * *}$ & 0,42 & 153 \\
\hline \multicolumn{4}{|l|}{ Alter des jüngsten Kindes } \\
\hline $0-2$ & $2,36^{\star * *}$ & $2,47^{\star \star}$ & 240 \\
\hline $3-5$ & 1,73 & 1,22 & 150 \\
\hline 6-17 (ref.) & 1 & 1 & 177 \\
\hline \multicolumn{4}{|l|}{ Bildung der Mutter } \\
\hline Niedrig & 1,03 & 1,45 & 179 \\
\hline Mittel (ref.) & 1 & 1 & 296 \\
\hline Hoch & 0,63 & 0,56 & 92 \\
\hline \multicolumn{4}{|l|}{ Jahr der Trennung } \\
\hline 1980-1989 (ref.) & 1 & 1 & 342 \\
\hline $1990-2000$ & 1,20 & $2,17^{* *}$ & 225 \\
\hline Pseudo R2 & 0,06 & & \\
\hline $\mathrm{n}$ & 567 & & \\
\hline $\mathrm{df}$ & 14 & & \\
\hline
\end{tabular}

Signifikanzniveaus: $* \mathrm{p}<0,10, * * \mathrm{p}<0,05, * * * \mathrm{p}<0,01$

${ }^{\text {a }}$ Wie Tabelle A1; die Variable Alter der Mutter wurde durch die Variable Alter des jüngsten Kindes ersetzt.

Quelle: Fertility and Family Survey 1995/96 und Generations and Gender Survey 2008/09 (eigene Berechnungen) 